\title{
An Inquiry into Two Intriguing Values of the Critical Current Density of Bi-2212
}

\author{
Gulshan Prakash Malik ${ }^{1}$, Vijaya Shankar Varma ${ }^{2}$ \\ ${ }^{1}$ B-208 Sushant Lok 1, Gurugram, India \\ ${ }^{2} 180$ Mall Apartments, Delhi, India \\ Email: gulshanpmalik@yahoo.com,varma2@gmail.com
}

How to cite this paper: Malik, G.P. and Varma, V.S. (2021) An Inquiry into Two Intriguing Values of the Critical Current Density of Bi-2212. World Journal of Condensed Matter Physics, 11, 53-64. https://doi.org/10.4236/wicmp.2021.113004

Received: July 14, 2021

Accepted: August 6, 2021

Published: August 9, 2021

Copyright $\odot 2021$ by author(s) and Scientific Research Publishing Inc. This work is licensed under the Creative Commons Attribution International License (CC BY 4.0).

http://creativecommons.org/licenses/by/4.0/

\section{(c) (i) Open Access}

\begin{abstract}
The empirically reported values of the critical current density $\left(j_{c}\right)$ of Bi-2212 as $2.4 \times 10^{5}\left(j_{c 1}\right.$; Sample 1$)$ and $1.0 \times 10^{6} \mathrm{~A} / \mathrm{cm}^{2}\left(j_{c}\right.$; Sample 2$)$ are intriguing because both of them correspond to the same values of the temperature $T=$ $4.2 \mathrm{~K}$ and the applied magnetic field $H=12 \times 10^{4} \mathrm{G}$. This difference is conventionally attributed to such factors-not all of which are quantifiable-as the geometry, dimensions and the nature of dopants and the manners of preparation of the samples which cause their granular structures, grain boundaries, alignment of the grains and so on to differ. Based on the premise that the chemical potential $\mu$ subsumes most of these features, given herein is a novel explanation of the said results in terms of the values of $\mu$ of the two samples. This paper revisits the problem that was originally addressed in [Malik G.P., Varma V.S. (2020) WJCMP, 10, 53-70] in the more accurate framework of a subsequent paper [Malik G.P., Varma V.S. (2021) JSNM, 34, 1551-1561]. Besides, it distinguishes between the contributions of the electro-electron (e-e) and the hole-hole $(h-h)$ pairs to $j_{c}$-a feature to which no heed was paid earlier. The essence of our findings is that the $j s$ of the two samples differ because they are characterized by different values of the primary variables $\mu_{i}$ and $\eta_{i} \equiv m_{i}^{*} / m_{e}$, where $m_{i}^{*}$ is the effective mass of a charge-carrier and $m_{e}$ is the free-electron mass and $i=1$ and 2 denote Sample 1 and Sample 2, respectively. In the scenario of the charge-carriers being predominantly $h-h$ pairs, the values of these parameters are estimated to be: $\mu_{1} \approx 12.3 \mathrm{meV}, \eta_{1} \approx 0.58 ; \mu_{2} \approx 22.7 \mathrm{meV}, \eta_{2} \approx 0.94$. Following from these and similar estimates when the charge-carriers are $e$-e pairs, given below for each sample are the detailed results for the values of the secondary variables viz. the number density of the charge-carriers and their critical velocity, the number of occupied Landau levels and the magnetic interaction parameter.
\end{abstract}

\section{Keywords}

Chemical Potential-, Temperature- and Applied Magnetic Field-Dependent 
Critical Current Density of Superconductors, Number Density, Landau

Quantization, Law of Equipartition of Energy, Bi-2212

\section{Introduction}

Among the family of Bi-based cuprates, $\mathrm{Bi}_{2} \mathrm{Sr}_{2} \mathrm{CaCu}_{2} \mathrm{O}_{8}(\mathrm{Bi}-2212)$ and $\mathrm{Bi}_{2} \mathrm{Sr}_{2} \mathrm{Ca}_{2} \mathrm{Cu}_{3} \mathrm{O}_{10}$ (Bi-2223) belong to the class of superconductors (SCs) that has the highest values of the critical temperature $\left(T_{c}\right)$, critical magnetic field $\left(H_{c}\right)$ and critical current density $\left(j_{c}\right)$. Although Bi-2223 is characterized by $T_{c} \approx 110 \mathrm{~K}$ and Bi-2212 by $T_{c} \approx 95 \mathrm{~K}$, the former-unlike the latter-has not only been found very hard to fabricate, but also almost impossible to obtain in a predominantly single phase. For these reasons, Bi-2212 is an SC that is among those that are most suitable for industrial applications and hence-given that as yet there is no consensus about the detailed mechanism that leads to high- $T_{c} s-$ it warrants that its superconducting features be probed from as many different approaches as possible.

Employing the formalism of the Bethe-Salpeter equation (BSE), we deal in this paper with two empirical values of $j_{c}(T, H)$, i.e., $2.4 \times 10^{5}$ and $1.0 \times 10^{6} \mathrm{~A} / \mathrm{cm}^{2}$ which are intriguing because they have been reported for the same values of $T$ and $H$, viz $T=4.2 \mathrm{~K}$ and $H=12 \times 10^{4} \mathrm{G}$ [1]. This is a problem that we had earlier addressed in [2]-Paper I hereafter, based on the novel premise that the chemical potential $(\mu)$ of an SC subsumes most of the features to which its widely varying values of $j_{c}(T, H)$ are conventionally attributed-features such as its geometry (wire, tape, thin film, etc.) and dimensions, the type of dopants it contains, and the manner of its preparation which causes different samples of it to be characterized by different granular structures and grain boundaries, alignment of the grains and so on, see e.g., [3], most of which are not quantifiable. Another remarkable feature of the approach followed in Paper I is that, unlike almost all conventional approaches, it employs directly the basic equation that defines $j_{c}(T, H)$ as the product of the electronic charge $e$, the number of superconducting charge-carriers $n_{s}(T, H)$ and their critical velocity $v_{c}(T, H)$.

The framework of Paper I comprised three core equations: (i) a $\mu$-, $T$ - and $H$-dependent pairing equation corresponding to $\boldsymbol{P}=0$ and hence $j_{c}=0$, where $\boldsymbol{P}$ denotes the 3-momentum of Cooper pairs in the lab frame, (ii) a $\mu$-, $T$ - and $H$-dependent pairing equation corresponding to $\boldsymbol{P} \neq 0$ and hence $j_{c} \neq 0$ and (iii) an equation relating Fermi energy $E_{F}$ with the number density of the chargecarriers $n_{s^{\prime}}$ It was pointed out in [4] - Paper II hereafter, which was devoted to a study of the variation of $j_{c}(T, H)$ with $T$ (for a fixed value of $H$ ) and $H$ (for a fixed value of $T$ ) that the framework of Paper I was deficient in that 1) the limits of both the $\boldsymbol{P}=0$ and the $\boldsymbol{P} \neq 0$ equations were incorrect for a reason that will be given below, 2) the assumption that $\boldsymbol{P}=0$ and $\boldsymbol{P} \neq 0$ equations are characterized by the same interaction parameter is unjustifiable and hence should be done away with and 3) it employed an equation for $n_{s}$ which, strictly speaking, is valid 
only when both $T=0$ and $H=0$, in lieu of which a more accurate $\mu$-, $T$ - and $H$-dependent number equation was employed in Paper II.

A feature that we did not pay heed to while dealing with $j_{c}(T, H)$ values of both the elemental and composite SCs in several papers (Refs. 6 - 11 in Paper II) is the relative contributions of the electron-electron $(e-e)$ and the hole-hole $(h-h)$ pairs to $j_{c}$. We deal here with the problem of the "intriguing" values of $j_{c}(T, H)$ of Bi-2212 in the enlarged framework of Paper II which now includes this feature.

The paper is organized as follows. Given in the next section is the theoretical framework employed in this study. In essence, it is a slightly enhanced framework of Paper II in that it now distinguishes between the two types of pairs as noted above. Dealt with in detail in Section 3 is the problem of the "intriguing" values of $j_{c}$. Sections 4 and 5, respectively, are devoted to a Discussion of our findings and the Conclusions following from them.

\section{Theoretical Framework}

Both in Paper I and Paper II, the $\mu$ - and $T$-incorporated BSE was further generalized to include $H$ applied in the $z$-direction via the following substitutions which constitute the Landau quantization prescription (LQP):

$$
\begin{gathered}
\int \mathrm{d} p_{x} \mathrm{~d} p_{y}=2 \pi e H \sum_{n}, \frac{p_{x}^{2}}{2 m^{*}}+\frac{p_{y}^{2}}{2 m^{*}}=(n+1 / 2) \hbar \Omega(H, \eta) \\
\left.\Omega_{0}=1.7588 \times 10^{7} \mathrm{rad} \cdot \mathrm{sec}^{-1} \cdot \mathrm{G}^{-1} \text { (for a particle of mass }=m_{e}\right) \\
\Omega(H, \eta)=\Omega_{0} H / \eta\left(\text { for a particle of mass }=m^{*}=\eta m_{e}\right)
\end{gathered}
$$

where $m^{*}$ is the effective mass of a charge carrier and $m_{e}$ the free electron mass. The upper limits of the integral and sum in (1) are usually infinity, which cannot be so in our problem because the energy of an electron/hole is constrained to lie between $\mu-k \theta$ and $\mu+k \theta$, where $k$ is the Boltzmann constant and $\theta$ the Debye temperature of the ions. Hence, its energy was divided between the $\left(p_{x}+p_{y}\right)$ and $p_{z}$ degrees of freedom by appealing to the law of equipartition of energy (LEE) as

$$
\begin{gathered}
(2 / 3)(\mu-k \theta) \leq\left(p_{x}^{2}+p_{y}^{2}\right) / 2 m^{*}=(n+1 / 2) \Omega\left(H_{c}, \eta\right) \leq(2 / 3)(\mu+k \theta) \\
(1 / 3)(\mu-k \theta) \leq p_{z}^{2} / 2 m_{1}^{*} \leq(1 / 3)(\mu+k \theta) .
\end{gathered}
$$

We note that in lieu of the second of the above relations, we had incorrectly employed $\left(-k \theta / 3 \leq p_{z}^{2} / 2 m^{*}-\mu \leq k \theta / 3\right)$ in Paper I.

Recapitulated below from Paper II are the equations which comprise our framework in this study. For the convenience of the reader, they have been written in terms of the units commonly employed in the BCS theory, e.g., Gauss for $H$, although they were derived by employing the natural system of units $\{e V, \hbar=1, c=1\}$.

\subsection{The Pairing Equation Incorporating $\mu, T$ and $H$ When $P=0$ and Hence $\boldsymbol{j}_{c}=0$}

Denoting the values of $j_{\mathcal{c}}$ the magnetic interaction parameter $\lambda_{m}, \mu, T_{\mathcal{o}} H_{c}$ and $\eta$ 
by $j_{c 0}, \lambda_{m 0}, \mu_{0}, T_{c 0}, H_{c 0}$ and $\eta_{0}$, respectively, when $j_{c}=0$, the equation for this case is

$$
\begin{aligned}
& 1=\lambda_{m 0} \int_{L_{1}}^{L_{2}} \frac{\mathrm{d} \xi}{\sqrt{1+\xi / \mu_{0}}} \\
& \times \sum_{n=0}^{n_{1}\left(\theta, \rho, H_{c 0}, \eta_{0}\right)} \frac{\tanh \left[\left(\Omega\left(H_{c 0}, \eta_{0}\right) / 2 k T_{c 0}\right)\left\{\xi / \Omega\left(H_{c 0}, \eta_{0}\right)+n+1 / 2\right\}\right]}{\Omega\left(H_{c 0}, \mu_{0}\right)\left[\xi / \Omega\left(H_{c 0}, \eta_{0}\right)+n+1 / 2\right]},
\end{aligned}
$$

where

$$
\begin{gathered}
\lambda_{m 0}=\frac{e H_{c 0} V}{16 \pi^{2}} \sqrt{\frac{2 \eta_{0} m_{e}}{\rho k \theta}} \\
L_{1}=-(2 \rho+1) k \theta / 3, L_{2}=(-2 \rho+1) k \theta / 3 \\
n_{1}\left(\theta, \rho, H_{c 0}, \eta_{0}\right)=\text { floor }\left[\frac{2(\rho+1) k \theta}{3 \Omega\left(H_{c 0}, \eta_{0}\right)}-1 / 2\right] \\
\mu_{0}=\rho k \theta
\end{gathered}
$$

and, as shown in Paper I, $\lambda_{m 0}$ is dimensionless and $V$ is the BCS interaction parameter as in $[N(0) V]$.

Remarks:

1) Equation (2) above is identical with Equation (5) of Paper I except that, because of the error in the application of LEE, the limits of the integral in the latter equation were from $-L_{1}(\theta)$ to $L_{1}(\theta)$ and the upper limit of the sum was $n_{m}\left(\theta, H_{c}\right)$ as given below

$$
L_{1}(\theta)=k \theta / 3, n_{m}\left(\theta, H_{c}\right)=\text { floor }\left\{\frac{2 k \theta}{3 \hbar \Omega_{1}\left(H_{c}\right)}-\frac{1}{2}\right\} .
$$

2) A more compact form of (2) given in Paper II that we employ here is

$$
\begin{aligned}
1= & 2 \lambda_{m 0} \int_{L_{3}(\rho)}^{L_{4}(\rho)} \mathrm{d} z \\
& \times \sum_{n=0}^{n_{1}\left(\theta, \rho, H_{c 1}, \eta_{1}\right)} \frac{\tanh \left[\left(\rho \theta / 2 T_{c 0}\right)\left\{z^{2}-1+(n+1 / 2) \Omega\left(H_{c 1}, \eta_{0}\right) / \rho k \theta\right\}\right]}{z^{2}-1+(n+1 / 2) \Omega\left(H_{c 0}, \eta_{0}\right) / \rho k \theta}
\end{aligned}
$$

where

$$
L_{3}(\rho)=\sqrt{(\rho-1) / 3 \rho}, L_{4}(\rho)=\sqrt{(\rho+1) / 3 \rho} .
$$

\subsection{The Pairing Equation Incorporating $\mu, T$ and $H$ When $P \neq 0$ and Hence $j_{c} \neq 0$}

For this case, in lieu of the (erroneous) Equation (17) in Paper I, we have the following compact equation where $\mu_{i}$ has been employed interchangeably with $q_{i} \mu_{0}$ or $q_{i} \rho k \theta$.

$$
1=\lambda_{m i} \int_{z_{L i}}^{z_{U_{i}}} \mathrm{~d} z \sum_{n=0}^{n_{L}} \frac{\tanh \left[A_{i}\right]+\tanh \left[B_{i}\right]}{z^{2}-1+(n+1 / 2) \hbar \Omega\left(H_{c i}, \eta_{i}\right) / \mu_{i}}, \quad(i=1 \text { or } 2)
$$

where 


$$
\begin{gathered}
\lambda_{m i}=\frac{H_{c i}}{H_{c 0}} \sqrt{\frac{\eta_{i} \mu_{0}}{\eta_{0} \mu_{i}}} \lambda_{m 0} \\
z_{L i}=\sqrt{1+\frac{-1-2 q_{i} \rho+1 / y_{i}}{3 q_{i} \rho}}, z_{U i}=\sqrt{1+\frac{1-2 q_{i} \rho-1 / y_{i}}{3 q_{i} \rho}} \\
n_{L i}=\text { floor }\left[\frac{2 k \theta}{3 \hbar \Omega\left(H_{c i}, \eta_{i}\right)}\left(1+q_{i} \rho-1 / y_{i}\right)-1 / 2\right] \\
\tanh \left[A_{i}\right]=\tanh \left[\left(q_{i} \rho \theta / 2 T_{c i}\right)\left\{z^{2}-1+(n+1 / 2) \Omega\left(H_{c i}, \eta_{i}\right) / q_{i} \rho k \theta+1 / q_{i} \rho y_{i}\right\}\right] \\
\left.B_{i}\right]=\tanh \left[\left(q_{i} \rho \theta / 2 T_{c i}\right)\left\{z^{2}-1+(n+1 / 2) \Omega\left(H_{c i}, \eta_{i}\right) / q_{i} \rho k \theta-1 / q_{i} \rho y_{i}\right\}\right] \\
y_{i}=\frac{k \theta}{\left|\boldsymbol{P}_{i}\right|} \sqrt{\frac{6 m_{i}^{*}}{\mu_{i}}}\left(\left|\boldsymbol{P}_{i}\right| \text { and } m_{1}^{*} \text { in units of electron-Volt }\right)
\end{gathered}
$$

and the subscript $i=1$ denotes that the symbol corresponds to $j_{c 1}$ and $i=2$ that it corresponds to $j_{c 2}$.

It is easily seen that when $T_{c i}=T_{c 0}, H_{c i}=H_{c 0}, \mu_{i}=\mu_{0}$ (i.e., $\left.q_{i}=1\right), \eta_{i}=\eta_{0}$ and $\boldsymbol{P}_{i}$ $=0$ (i.e., $\left.y_{i}=\infty\right)$, (4) reduces to (3) as it ought to.

Equation (4) does not shed light on the relative contributions of the $e$-e and the $h-h$ pairs to it, which is an additional feature that we are now interested in. This is a situation that is easily remedied by revisiting the derivation of (4), the starting point of which was Equation (9) in Paper II in which the limits of the integral (say $F$ ) were from

$$
(\mu-k \theta+\alpha) / 3 \text { to }(\mu+k \theta-\alpha) / 3\left(\alpha=|\boldsymbol{P}| \sqrt{\mu / 6 m^{*} c^{2}}\right) .
$$

If we split these limits into two parts as from $(\mu-k \theta+\alpha) / 3$ to $\mu / 3$ and from $\mu / 3$ to $(\mu+k \theta-\alpha) / 3$, change the variable of integration from $p_{z}$ to $\xi=p_{z}^{2} / 2 m-\mu / 3$ and follow it up just as we did in Paper II, then $F$ is also split into two parts and in lieu of (4) we obtain

$$
1=F_{1 i}\left(\lambda_{m 0}, \theta, \rho, \mu_{i}, T_{c 1}, H_{c i}, y_{i}\right)+F_{2 i}\left(\lambda_{m 0}, \theta, \rho, \mu_{i}, T_{c 1}, H_{c i}, y_{i}\right),
$$

where

$$
\begin{aligned}
& F_{1 i}\left(\lambda_{m 0}, \theta, \rho, \mu_{i}, T_{c 1}, H_{c i}, y_{i}\right) \\
& =\lambda_{m i} \int_{L_{i}\left(\rho, \mu_{i}, y_{i}\right)}^{1 / \sqrt{3}} \mathrm{~d} z \sum_{n=0}^{n_{L}} \frac{\tanh \left[A_{i}\right]+\tanh \left[B_{i}\right]}{z^{2}-1+(n+1 / 2) \hbar \Omega\left(H_{c i}, \eta_{i}\right) / q_{i} \rho k \theta} \\
& F_{2 i}\left(\lambda_{m 0}, \theta, \rho, \mu_{i}, T_{c 1}, H_{c i}, y_{i}\right) \\
& =\lambda_{m i} \int_{1 / \sqrt{3}}^{U_{i}\left(\rho, \mu_{i}, y\right)} \mathrm{d} z \sum_{n=0}^{n_{L}} \frac{\tanh \left[A_{i}\right]+\tanh \left[B_{i}\right]}{z^{2}-1+(n+1 / 2) \hbar \Omega\left(H_{c i}, \eta_{i}\right) / q_{i} \rho k \theta},
\end{aligned}
$$

and

$$
L_{i}\left(\rho, \mu_{i}, y_{i}\right)=\operatorname{Re}\left[\sqrt{\frac{1}{3}-\frac{1-1 / y_{i}}{3 \mu_{i} / k \theta}}\right], U_{i}\left(\rho, \mu_{i}, y_{i}\right)=\left[\sqrt{\frac{1}{3}+\frac{1-1 / y_{i}}{3 \mu_{i} / k \theta}}\right]
$$

When (8) is solved in conjunction with the number equation given below, (9) 
and (10), respectively, give the desired relative contributions of the $h-h$ and the $e$-e pairs to $j_{c 1}$ when $i=1$ and to $j_{c 2}$ when $i=2$.

\subsection{The $T$ - and $H$-Incorporated Number Equation}

In lieu of the naïve equation employed in Paper I

$$
n_{s}\left(E_{F}\right)=\frac{1}{3 \pi^{2}}\left[\frac{2 m^{*} E_{F}}{(\hbar c)^{2}}\right]^{3 / 2}\left(m^{*} \text { and } E_{F} \text { in units of electron-Volt }\right),
$$

the number equation employed in Paper II is

$$
\begin{aligned}
& n_{s i}\left(\theta, \mu_{i}, T_{c i}, H_{c i}, \eta_{i}\right)=2 P_{F}\left(H_{c i}\right) \int_{0}^{x_{U}} \mathrm{~d} x \\
& \times \sum_{n=0}^{n_{m}\left(\theta, \mu_{i}, H_{c i}, \eta_{i}\right)}\left[1-\tanh \left\{\frac{\hbar \Omega\left(H_{c i}, \eta_{i}\right)}{2 k T_{c i}}\left[n+1 / 2+x^{2}-\frac{\mu_{i}}{\hbar \Omega\left(H_{c i}, \eta_{i}\right)}\right]\right\},\right.
\end{aligned}
$$

where

$$
\begin{gathered}
P_{F}\left(H_{c i}\right)=\frac{b_{1} b_{2} H_{c i}}{4 \sqrt{2} \pi^{2}} \sqrt{\frac{m_{e} c^{2} \Omega_{0} H_{c i}}{a_{3}}} \frac{1}{(\hbar c)^{3}}, x_{U}=\sqrt{\frac{\mu_{i}+k \theta}{3 \hbar \Omega\left(H_{c i}, \eta_{i}\right)}} \\
b_{1}=(137.0359895)^{-1 / 2}, b_{2}=6.9250774 \times 10^{-2}, a_{3}=1.51926689 \times 10^{15} \\
n_{m}\left(\theta, \mu_{i}, H_{c i}, \eta\right)=\text { floor }\left[\frac{2\left(k \theta+\mu_{i}\right)}{3 \hbar \Omega\left(H_{c i}, \eta_{i}\right)}-1 / 2\right] .
\end{gathered}
$$

Equation (11) was derived in [5].

From the definitions of $y_{i}$ given below (4) and $\left|\boldsymbol{P}_{c i}\right|=2 m_{i}^{*}\left|\boldsymbol{v}_{c i}\right|$, it follows that

$$
\left|\boldsymbol{v}_{c i}\left(\theta, \rho, q_{i}, \eta_{i}, y_{i}\right)\right|=\frac{c}{2 y_{i}} \sqrt{\frac{6 k \theta}{q_{i} \rho \eta_{i}\left(m_{e} c^{2}\right)}},
$$

whence substituting (11) and (12) into $j_{c i}\left(T_{c i}, H_{c i}\right)=e n_{s i}\left(T_{c i}, H_{c i}\right) v_{c i}\left(T_{c i}, H_{c i}\right)$, we obtain

$$
1-\frac{j_{c i}\left(T_{c i}, H_{c i}\right)}{e n_{s i}\left(\theta, q_{i}, T_{c i}, H_{c i}, \eta_{i}\right)\left|v_{c i}\left(\theta, \rho, q_{i}, \eta_{i}, y_{i}\right)\right|}=0 .
$$

\section{Dealing with the "Intriguing" Values of $j_{c}$ of Bi-2212}

To deal with the "intriguing" values of $j_{c}$ of $\mathrm{Bi}-2212$, we also need empirical data corresponding to the $j_{c}=0$ situation in order to fix the interaction parameters when $j_{c} \neq 0$. Assuming as in Paper II that the pairing mechanism is the 1-phonon exchange mechanism due to Ca ions, given below are the empirical data [1] that we have employed for both these cases in our study.

$$
\begin{gathered}
\theta(\text { Ca ions })=237 \mathrm{~K} \\
T_{c 0}=65 \mathrm{~K}, H_{c 0}=36 \times 10^{4} \mathrm{G}, j_{c 0}=0 \\
T_{c 1}=4.2 \mathrm{~K}, H_{c 1}=12 \times 10^{4} \mathrm{G}, j_{c 1}=2.4 \times 10^{5} \mathrm{~A} / \mathrm{cm}^{2} \\
T_{c 2}=T_{c 1}, H_{c 2}=H_{c 1}, j_{c 2}=1.0 \times 10^{6} \mathrm{~A} / \mathrm{cm}^{2} .
\end{gathered}
$$


Taking stock of the unknown parameters in our problem, we find that we have five of them viz., $\rho$ (or $\mu_{0}$ ), $\eta_{0}, q_{i}$ (or $\mu_{i}$ ), $\eta_{i}$ and $y_{i}$ (because fixing $\rho$ and $\eta_{0}$ fixes $\lambda_{m 0}$, whence $\lambda_{m i}$ too is fixed), whereas the number of equations at our disposal is three viz. (2), (8) and (13). It follows that we need to assume the values of two of these parameters in order to calculate the rest of them. Based on diverse criteria, we give below a template for the values of various parameters and demand that both types of parameters conform to it.

\subsection{A Template for the Values of Various Parameters Associated with $\boldsymbol{j}_{c 0}, \boldsymbol{j}_{c 1}$ and $\boldsymbol{j}_{c 2}$}

Guided by the current wisdom about the values of some select parameters in the superconductivity of high- $T_{c} \mathrm{SCs}$, such as:

1) they are characterized by much lower values of the Fermi energy or the chemical potential than the elemental SCs for which it is of the order of $1-10 \mathrm{eV}$;

2) the effective mass of electrons in one of them, viz., $\mathrm{MgB}_{2}$, has been estimated to be $0.44-0.68$ [6];

3) the number density of charge-carriers $\left(n_{s}\right)$ in these SCs is several orders of magnitude smaller than for the elemental SCs for which it is of the order of $1.0 \times$ $10^{21}-10^{22} \mathrm{~cm}^{-3}$; and

4) the charge-carriers in some of them are e-e pairs, while for some others they are $h-h$ pairs, we adopt approximately the following values as a template in this study.

a) $\mu_{0}, \mu_{1}, \mu_{2}<200 \mathrm{meV}$

b) $0.4<\eta_{0}, \eta_{1}, \eta_{2}<1.4$

c) $n_{s 1}, n_{s 2}<5 \times 10^{18} \mathrm{~cm}^{-3}$

d) $\lambda_{m 00}, \lambda_{m 1}, \lambda_{m 2}<0.5$ (Bogoliubov constraint)

e) $F_{1}>50 \%\left(F_{2}>50 \%\right)$ for $j_{c i}$ to be regarded as predominantly due to $h-h(e-e)$ pairs.

\subsection{Procedure and Results}

Our procedure comprises the following steps:

1) Solve the core equation (2) for $\lambda_{m 0}$ with the input of the empirical values given in (14) and some select values of $\left\{\mu_{0}=\rho k \theta, \eta_{0}\right\}$ that are subject to the constraints in Sec 3.1, para 4). Corresponding to the choice of each such pair of values, we obtain a triplet of $\left\{\mu_{0}, \eta_{0}, \lambda_{m 0}\right\}$ values which is a requisite input for the simultaneous solution of the other two of our core equations viz. (8) and (13).

2) The above step implies that one has on command an innumerable number of the triplets of $\left\{\mu_{0}, \eta_{0}, \lambda_{m 0}\right\}$ for the requisite input as noted above because an innumerable number of them can be found that satisfy the requisite constraints. Nonetheless, we found that the empirically verifiable values of some of the parameters associated with $j_{c 1}$ or $j_{c 2}$ can be obtained via a consideration of just a few of these triplets.

3) As an illustration of the above remark, we give below-both for $j_{c 1}$ and 
$j_{c 2}$-the results for the following three sets of $\left\{\mu_{0}, \eta_{0}, \lambda_{m 0}\right\}$ :
a) $S_{1}\left(\mu_{0}, \eta_{0}, \lambda_{m 0}\right)=\left\{102 \mathrm{meV}(\rho=5), 0.80,5.8443 \times 10^{-2}\right\}$
b) $S_{2}\left(\mu_{0}, \eta_{0}, \lambda_{m 0}\right)=\left\{153 \mathrm{meV}(\rho=7.5), 0.60,6.97801 \times 10^{-2}\right\}$
c) $S_{3}\left(\mu_{0}, \eta_{0}, \lambda_{m 0}\right)=\left\{204 \mathrm{meV}(\rho=10), 1.0,4.03497 \times 10^{-2}\right\}$.

4) Given in Table 1 are the detailed results corresponding to $\left\{j_{c 1}, S_{1}\right\}$.

5) Although we have obtained results corresponding to $\left\{j_{c 1}, S_{2}\right\},\left\{j_{c 1}, S_{3}\right\},\left\{j_{c_{2}}\right.$, $\left.S_{1}\right\},\left\{j_{c 2}, S_{2}\right\}$ and $\left\{j_{c 2}, S_{3}\right\}$ in similar detail as given in Table 1, for the sake of brevity we summarize in Table 2 the results for all the six sets in the form of widths which are determined as follows. As is seen from Table 1 corresponding to $\left\{j_{c}\right.$, $\left.S_{1}\right\}$ that when the charge-carriers are e-e pairs, $\mu_{1}$ varies between 1.79 and 5.0 $\mathrm{meV}$, i.e., its width is $(1.79-5.0) \mathrm{meV}$. Similar widths corresponding to $\left\{j_{c 1}, S_{2}\right\}$ and $\left\{j_{j c 1}, S_{3}\right\}$, respectively, are $(1.37-4.60) \mathrm{meV}$ and $(1.80-5.0) \mathrm{meV}$. The width quoted for $\mu_{1}$ in Table 2 corresponding to all three of these cases is (1.37 - 5.0) $\mathrm{meV}$, which is greater than any of them individually and hence is a liberal estimate. Widths (or bounds) of all the other parameters in Table 2 have been similarly obtained.

Table 1. Treating $\mu_{1}$ as the independent variable, values of $\eta_{1}$ and $y_{1}$ obtained by solving (8) and (13) with the inputs of $S_{1}\left(\mu_{0}, \eta_{0}, \lambda_{m 0}\right)$ and the empirical values given in (15). The values of $n_{s 1}, V_{c 1}, \lambda_{m 1}, n_{L 1}, F_{11}$ and $F_{21}$ are determined via (11), (12), (5), (6), (9) and (10), respectively. $\mu_{1}$ is restricted as $1.79 \leq \mu_{1} \leq 16.0 \mathrm{meV}$ because outside of this range one or the other constraints noted in Section 3.1 is violated.

\begin{tabular}{|c|c|c|c|c|c|c|c|}
\hline$\mu_{1} \mathrm{meV}$ & $\eta_{1}$ & $y_{1}$ & $n_{s 1} \times 10^{17}$ & $V_{c} \times 10^{6}$ & $\lambda_{m 1}, n_{L 1}$ & $F_{11}, F_{21}(\%)$ & $\begin{array}{l}\text { Charge } \\
\text { carriers }\end{array}$ \\
\hline 1.79 & 1.3850 & 4.1121 & 2.92 & 5.12 & $1.9360 \mathrm{e}-1,10$ & $26.0,74.0$ & \multirow{6}{*}{$e$-e pairs } \\
\hline 1.90 & 1.3292 & 4.1670 & 2.99 & 5.01 & $1.8409 \mathrm{e}-1,10$ & $27.2,72.8$ & \\
\hline 2.10 & 1.2441 & 4.2795 & 3.12 & 4.80 & $2.6941 \mathrm{e}-1,10$ & $29.1,70.9$ & \\
\hline 3.10 & 1.0470 & 5.2074 & 4.24 & 3.54 & $1.2791 \mathrm{e}-1,9$ & $34.8,65.2$ & \\
\hline 4.20 & 0.9155 & 6.1608 & 5.46 & 2.75 & $1.0276 \mathrm{e}-1,8$ & $42.1,57.9$ & \\
\hline 5.00 & 0.8063 & 6.4566 & 5.86 & 2.56 & $8.8386 \mathrm{e}-2,8$ & $48.6,51.4$ & \\
\hline 6.00 & 0.7434 & 7.0853 & 6.76 & 2.22 & $7.7470 \mathrm{e}-2,7$ & $54.0,46.0$ & \\
\hline 7.00 & 0.6649 & 7.3592 & 7.17 & 2.09 & $6.7833 \mathrm{e}-2,7$ & $57.7,42.3$ & \\
\hline 7.90 & 0.6115 & 7.6365 & 7.58 & 1.98 & $6.1234 \mathrm{e}-2,7$ & $60.0,40.0$ & \\
\hline 9.10 & 0.5757 & 8.3758 & 8.66 & 1.73 & $5.5358 \mathrm{e}-2,6$ & $62.1,37.9$ & \\
\hline 10.0 & 0.5398 & 8.6423 & 9.07 & 1.65 & $5.1138 \mathrm{e}-2,6$ & $63.2,36.8$ & \\
\hline 11.0 & 0.5111 & 8.9621 & 9.60 & 1.56 & $4.7442 \mathrm{e}-2,6$ & $63.7,36.3$ & $h-h$ pairs \\
\hline 12.0 & 0.4850 & 9.2014 & 10.0 & 1.49 & $4.4248 \mathrm{e}-2,6$ & $64.6,35.4$ & \\
\hline 13.0 & 0.4595 & 9.3576 & 10.3 & 1.45 & $4.1377 e-2,6$ & $66.0,34.0$ & \\
\hline 14.0 & 0.4373 & 9.5142 & 10.6 & 1.41 & $3.8897 e-2,6$ & $67.2,32.8$ & \\
\hline 15.0 & 0.4194 & 9.7573 & 11.1 & 1.36 & $3.6804 \mathrm{e}-2,6$ & $68.2,31.8$ & \\
\hline 16.0 & 0.4050 & 10.083 & 11.6 & 1.29 & $3.5019 \mathrm{e}-2,6$ & $68.9,31.1$ & \\
\hline
\end{tabular}


Table 2. Following from the sets $\left\{j_{c 1}, S_{1}\right\},\left\{j_{c 1}, S_{2}\right\},\left\{j_{c 1}, S_{3}\right\},\left\{j_{c 2}, S_{1}\right\},\left\{j_{c 2}, S_{2}\right\}$ and $\left\{j_{c 2}, S_{3}\right\}$, the widths of the values of various parameters associated with $j_{c 1}$ and $j_{c 2}$.

\begin{tabular}{|c|c|c|c|c|c|c|c|}
\hline \multicolumn{8}{|c|}{$j_{c 1}=2.4 \times 10^{5} \mathrm{~A} / \mathrm{cm}^{2}$} \\
\hline Charge-carriers & $\mu_{1} \mathrm{meV}$ & $\eta_{1}$ & $y_{1}$ & $n_{s 1} \times 10^{17} \mathrm{~cm}^{-3}$ & $v_{c 1} \times 10^{6} \mathrm{~cm} / \mathrm{s}$ & $n_{L 1}$ & $\lambda_{m 1} \times 10^{-1}$ \\
\hline e-e pairs & $1.37-5.00$ & $1.4062-0.7363$ & $3.2763-6.9557$ & $2.05-6.56$ & $7.31-2.28$ & $11-6$ & $3.7590-0.8028$ \\
\hline$h-h$ pairs & $5.50-19.10$ & $0.7654-0.4030$ & $5.5063-12.1199$ & $4.64-15.5$ & $3.23-0.973$ & $8-5$ & $1.2617-0.2837$ \\
\hline \multicolumn{8}{|c|}{$j_{c 2}=1.0 \times 10^{6} \mathrm{~A} / \mathrm{cm}^{2}$} \\
\hline Charge-carriers & $\mu_{2} \mathrm{meV}$ & $\eta_{2}$ & $y_{2}$ & $n_{s 2} \times 10^{18} \mathrm{~cm}^{-3}$ & $V_{c 2} \times 10^{6} \mathrm{~cm} / \mathrm{s}$ & $n_{L 2}$ & $\lambda_{m 2} \times 10^{-1}$ \\
\hline$e-e$ pairs & $5.75-7.10$ & $1.4297-1.2203$ & $3.1506-3.7115$ & $1.70-2.19$ & 3.672 .87 & $14-12$ & $1.8531-0.9750$ \\
\hline$h$ - $h$ pairs & $7.40-38.0$ & $1.4000-0.4888$ & $3.5106-6.3018$ & $2.00-5.11$ & 3.121 .22 & $14-11$ & $1.3904-0.3289$ \\
\hline
\end{tabular}

\section{Discussion}

1) A feature of the study reported here that is worth drawing attention to is that, unlike the $T_{c} s$ and the gaps of $\mathrm{Bi}-2212$ which require 2- and 3-phonon exchange mechanisms [7], we needed here only the one-phonon exchange mechanism. This of course is due to the fact that putting an SC in a magnetic field considerably weakens the interaction parameter.

2) Although the role of the $e$-e and the $h$ - $h$ pairs in the original BCS theory has been a matter of debate in some quarters, it has been shown [8] that both types of pairs make equal contributions to the pairing amplitude when $T=0, H$ $=0$ and $E_{F} \gg k \theta$. The fact that we identified the pairs below the chemical potential surface as $h-h$ pairs and those above it as e-e pairs is because, as has also been noted by Ziman [9], "the excitations of the superconducting state are peculiar quasi-particles which change from being 'electrons' to being 'holes' as they pass through the Fermi level". Since we are now dealing with the situation where $T \neq 0, H \neq 0$ via LQP (which brings the effective mass of the charge-carriers into play) and values of $\mu$ that are not $\gg k \theta$, unsurprisingly, we have found that the two types of carriers, in general, do not make equal contributions. What seems remarkable is that we can quantify the contributions of such pairs in the mean-field approximation, i.e., without taking recourse to a Hamiltonian that has explicit terms corresponding to the $e-e$ and the $h-h$ pairs-which is an approach that been advocated by Llano and collaborators in several papers as, e.g., [10].

3) In dealing with $j_{c 1}$ subject to the constraints noted in Section 3.1, we have found that the values of $\mu_{1}$ and $n_{s 1}$-which may be empirically verifiable-corresponding to $S_{1}, S_{2}$ and $S_{3}$, respectively, are:

i) $\mu_{1}<16.0,11.0,19.1(\mathrm{meV})$

ii) $n_{s 1} \leq 11.6,6.65,15.4\left(\times 10^{17} \mathrm{~cm}^{-3}\right)$

4) If it is empirically found that the charge-carriers corresponding to $j_{c 1}$ are predominantly $h-h$ pairs, then the widths of the values of $\mu_{1}$ and $n_{s 1}$, corresponding to $S_{1}, S_{2}$ and $S_{3}$, respectively, are further narrowed down to

i) $\mu_{1}(\mathrm{meV}):(6.0-16.0),(7.0-11.0),(6.0-19.1)$ 
ii) $n_{s}\left(\times 10^{17} \mathrm{~cm}^{-3}\right)$ : $(6.76-11.6),(5.63-6.65),(7.05-15.4)$

5) If the charge carriers are predominantly $h-h$ pairs, then the widths of the values $\mu_{2}$ and $n_{s 2}$ similar to those in 3) and corresponding to $\left\{j_{c 2}, S_{1}\right\},\left\{j_{c 2}, S_{2}\right\}$ and $\left\{j_{c_{2}}, S_{3}\right)$, respectively, are:

i) $\mu_{2}(\mathrm{meV}):(7.80$ - 27.0), $(8.10-38.0),(7.40-24.5)$

ii) $n_{s 2}\left(\times 10^{18} \mathrm{~cm}^{-3}\right)$ : $(2.35-4.94),(2.10-5.11),(2.40-4.95)$.

5) Corresponding to the values of $\mu_{1}$ and $\mu_{2}$ in 3) and 4) above, the widths of the values of $\eta_{1}$ and $\eta_{2}$, respectively, are: (0.7434-0.4050), (1.4015 - 0.4030), (0.7654 - 0.4146) and (1.3105 - 0.6553), (1.1337 - 0.4888), (1.4000 - 0.7178).

6) While both $\mu_{1}$ and $\mu_{2}$ have values that are significantly lower than the values for the elemental SCs, $\mu_{2}>\mu_{1}$.

i) Unsurprisingly, $n_{s 2}>n_{s 1}$ and (mostly) $v_{c 2}<v_{c 1}$.

ii) While $\eta_{1}$ decreases as $\mu_{1}$ is increased, as does $\eta_{2}$ when $\mu_{2}$ is increased, quite generally, $\eta_{2}>\eta_{1}$, implying that $j_{c 2}$ is due to heavier charge carriers, in the context of which it seems relevant to point out that one is dealing here with a non-equilibrium state when the system is subject to an external stimulus.

\section{Conclusions}

It was noted in the Introduction above that there is as yet no consensus about the cause of the occurrence of high- $T_{c}$ superconductivity although, as expounded in, e.g. [11], a wide variety of mechanisms or models-such as the NambuEliashberg-McMillan extension of the BCS theory, non-phononic mechanisms invoking magnons (the Hubbard model, the $t$-J model), the RVB model, electronic models invoking excitons, and so on-has been proposed to account for it. Given such a situation, it is warranted that due heed be paid to the insights that all these approaches provide. Obtained via the BSE-based approach, we deem it plausible that the results reported in this paper such as the values of the chemical potential and the effective masses of the charge-carriers corresponding to the $j_{c 0}, j_{c 1}$ and $j_{c 2}$ may aid in reaching the goal of the final theory of high- $T_{c}$ superconductivity.

Since, as was noted above, there is an innumerable number of the triplets of $\left(\mu_{0}, \eta_{0}, \lambda_{m 0}\right)$ which can be employed for determining the values of various parameters associated with $j_{c i}$ it is remarkable that both the values of $\mu_{i}$ and $n_{s i}$ corresponding to $j_{c i}(i=1$ or 2$)$ fall in nearly the same ballpark for each of the three triplets $S_{1}, S_{2}$ and $S_{3}$ that we have employed. This implies that the values of various parameters corresponding to $j_{c i}$-not restricted to $\mu_{i}$ and $n_{s i}$-depend only mildly on the triplets $S_{1}-S_{3}$ employed to obtain them and that by appealing to just a few of them one is led to better than an order-of-magnitude estimate of these parameters.

If the width of each parameter in Table 2 is replaced by its mid-point, which is not unreasonable, then the values of some of the parameters that distinguish $j_{c 1}$ and $j_{c 2}$ when the charge-carriers are predominantly $h-h$ pairs are as follows:

Parameters characterizing $j_{c c}$ : 


$$
\mu_{1}=12.3 \mathrm{meV}, \eta_{1}=0.584, n_{s 1}=1.01 \times 10^{18} \mathrm{~cm}^{-3} .
$$

Parameters characterizing $j_{c 2}$ :

$$
\mu_{2}=22.7 \mathrm{meV}, \eta_{2}=0.944, n_{s 2}=3.55 \times 10^{18} \mathrm{~cm}^{-3} .
$$

It is gratifying to note that our suggestion that regardless of the physical attributes of an SC, its Fermi energy (i.e., $\mu$ at $T=0$ ) plays an important role in determining its properties is being taken note of, as evidenced by [12].

We conclude by drawing attention to [13] for an exposition of the BSE-based approach to superconductivity.

\section{Conflicts of Interest}

The authors declare no conflicts of interest regarding the publication of this paper.

\section{References}

[1] Poole, C.P. (2000) Handbook of Superconductivity. Academic Press, San Diego.

[2] Malik, G.P. and Varma, V.S. (2020) Fermi Energy-Incorporated Framework for Dealing with the Temperature- and Magnetic Field-Dependent Critical Current Densities of Superconductors and Its Application to Bi-2212. World Journal of Condensed Matter Physics, 10, 53-70. https://doi.org/10.4236/wjcmp.2020.102004

[3] Kumar, J., Sharma, D., Ahluwalia, P.K. and Awana, V.P.S. (2013) Enhanced Superconducting Performance of Melt Quenched $\mathrm{Bi}_{2} \mathrm{Sr}_{2} \mathrm{CaCu}_{2} \mathrm{O}_{8}$ (Bi-2212). Materials Chemistry and Physics, 139, 681-688.

https://doi.org/10.1016/j.matchemphys.2013.02.016

[4] Malik, G.P. and Varma, V.S. (2021) A New Microscopic Approach to Deal with the Temperature- and Applied Magnetic Field-Dependent Critical Current Densities of Superconductors. Journal of Superconductivity and Novel Magnetism, 34, 1551-1561. https://doi.org/10.1007/s10948-021-05852-8

[5] Malik, G.P. and Varma, V.S. (2020) On a New Number Equation Incorporating Both Temperature and Applied Magnetic Field and Its Application to $\mathrm{MgB}_{2}$. Journal of Superconductivity and Novel Magnetism, 33, 3681-3685. https://doi.org/10.1007/s10948-020-05639-3

[6] Yelland, E.A., et al. (2002) de Haas-van Alphen Effect in Single Crystal $\mathrm{MgB}_{2}$. Physical Review Letters, 88, Article ID: 217002.

[7] Malik, G.P. and Varma, V.S. (2019) A Generalized BCS Equations: A Review and a Detailed Study of the Superconducting Features of $\mathrm{Ba}_{2} \mathrm{Sr}_{2} \mathrm{CaCu}_{2} \mathrm{O}_{8}$. In: Superconductivity and Superfluidity, IntechOpen, London.

[8] Malik, G.P. (2008) High-T ${ }_{c}$ Superconductivity via Superpropagators Revisited. Physica $C$, 468, 949-954. https://doi.org/10.1016/j.physc.2008.03.002

[9] Ziman, J.M. (1972) Principles of the Theory of Solids. 2nd Edition, Cambridge University Press, Cambridge. https://doi.org/10.1017/CBO9781139644075

[10] Chávez, I., Grether, M. and de Llano, M. (2016) Generalized BEC and Crossover Theories of Superconductors and Ultracold Bosonic and Fermionic Gases. Journal of Superconductivity and Novel Magnetism, 29, 691-695.

https://doi.org/10.1007/s10948-015-3288-y

[11] Kresin, V.Z., Morawitz, H. and Wolf, S.A. (1993) Mechanisms of Conventional and 
High-Tc Superconductivity. Oxford University Press, New York.

[12] Mackinnon, I.D.R., Almutairi, A. and Alarco, J.A. (2021) Insights from Systematic DFT Calculations on Superconductors. In: Real Perspective of Fourier Transforms and Current Developments in Superconductivity, IntechOpen, London. https://doi.org/10.5772/intechopen.96960

[13] Malik, G.P. (2016) Superconductivity: A New Approach Based on the Bethe-Salpeter Equation in the Mean-Field Approximation. In: The Series on Directions in Condensed Matter Physics. World Scientific, Singapore, Volume 21.

https://doi.org/10.1142/9868 